TRANSACTIONS OF THE

AMERICAN MATHEMATICAL SOCIETY

Volume 355, Number 2, Pages 735-745

S 0002-9947(02)03127-6

Article electronically published on October 1, 2002

\title{
AXIOM A FLOWS WITH A TRANSVERSE TORUS
}

\author{
C. A. MORALES
}

\begin{abstract}
Let $X$ be an Axiom A flow with a transverse torus $T$ exhibiting a unique orbit $O$ that does not intersect $T$. Suppose that there is no nullhomotopic closed curve in $T$ contained in either the stable or unstable set of $O$. Then we show that $X$ has either an attracting periodic orbit or a repelling periodic orbit or is transitive. In particular, an Anosov flow with a transverse torus is transitive if it has a unique periodic orbit that does not intersect the torus.
\end{abstract}

\section{INTRODUCTION}

We shall consider Axiom A flows with a transverse torus on closed 3-manifolds. Examples of such flows are the suspension of a toral Axiom A diffeomorphism and the Anosov flows in $\mathrm{BL}, \overline{\mathrm{Br} 2}$, [FW], $\mathrm{FT}$ (see also Br1]). We give sufficient conditions for the transitivity of those flows based on the following definition. Let $S$ and $O$ be, respectively, a transverse surface and an orbit of a flow $X$. We say that $O$ is essential for $S$ if there is no null-homotopic closed curve in $S$ contained in either the stable set or the unstable set of $O$.

Theorem. Let $X$ be an Axiom A flow with a transverse torus $T$ exhibiting a unique orbit $O$ that does not intersect $T$. If $O$ is essential for $T$, then $X$ has either an attracting periodic orbit or a repelling periodic orbit or is transitive.

The Theorem can be used to study Anosov flows with a transverse torus on closed 3-manifolds. Indeed, the most classical examples of Anosov flows with a transverse torus are the suspended ones. Such examples motivated Barbot and Ghys to conjecture that the existence of a transverse torus suffices for a transitive Anosov flow to be suspended. This conjecture was proved to be false in $[\mathrm{BL}],[\mathrm{Br} 2]$. In particular, BL exhibited an Anosov flow with a transverse torus that is transitive (because it has an invariant volume form) and not suspended (because it has a unique periodic orbit that does not intersect the torus). The corollary below asserts that, in general, the transitivity follows from the existence of a unique periodic orbit that does not intersect the torus. More precisely, we have the following result.

Corollary. An Anosov flow with a transverse torus on a closed 3-manifold is transitive if it has a unique periodic orbit that does not intersect the torus.

Let us explain the main ideas behind the proof of our results. The foliation theory [HH] has been playing a fundamental role in the study of Anosov flows on closed

Received by the editors October 8, 2001 and, in revised form, February 7, 2002.

2000 Mathematics Subject Classification. Primary 37D20; Secondary 37E99.

Key words and phrases. Anosov flow, Axiom A flow, transverse torus.

The author was partially supported by FAPERJ, CNPq and PRONEX-Brasil. 
3-manifolds. This is because the weak stable (unstable) manifolds of the Anosov flows form codimension-one foliation in the ambient manifold. Now, the weak stable manifolds of the Axiom A flows do not form, in general, a foliation (even if the flow is nonsingular and has neither attracting periodic orbits nor repelling periodic orbits). So, the foliation theory cannot be applied in our context. To overcome this problem, we shall consider the return map $\Pi$ associated to the transverse torus $T$. Briefly the proof goes as follows: Let $X, O$ and $T$ be as in the statement of the Theorem. Assume that $X$ has neither attracting periodic orbits nor repelling periodic orbits. By Proposition 3.6, we have that $O$ is a hyperbolic saddle-type periodic orbit of $X$. So, there are two (either equal or disjoint) simple closed curves $C_{1}^{s}, C_{2}^{s} \subset T$ in the stable manifold of $O$ so that the domain of $\Pi$ is $T \backslash\left(C_{1}^{s} \cup C_{2}^{s}\right)$. If $X$ were not transitive, then there would exist (after reversing the flow if necessary) a hyperbolic strange attractor $\Lambda$ that does not contain $O$. In particular, $\Lambda \cap\left(C_{1}^{s} \cup C_{2}^{s}\right)=\emptyset$. Denoting $\Lambda^{*}=\Lambda \cap T$, we would have that $\Lambda^{*}$ is a hyperbolic strange attractor of $\Pi$. Since $O$ is essential by assumption, we have that $C_{1}^{s}$ is not null-homotopic in $T$. Then, the surface $A$ obtained by cutting $T$ open along $C_{1}^{s}$ is an annulus which clearly embeds into $S^{2}$. Since $A$ embeds into $S^{2}$, we have that $\Lambda^{*}$ is a hyperbolic strange attractor in $S^{2}$. By Lemma 3.1, we shall find a local basin of attraction $U^{*} \subset A$ of $\Lambda^{*}$ such that the image of the boundary curves of $U^{*}$ under $\Pi$ are not null-homotopic in $U^{*}$. By $\left[\mathbb{R}\right.$, Theorem 9.1, p. 304] we have that $U^{*}$ has at least three holes. Since $A$ is an annulus, it would follow that one of these holes bounds a disk $D$ in the domain of $\Pi$. Since $U$ has finitely many holes, we will find an iterated $\Pi^{n}$ of $\Pi$ such that $D \subset \operatorname{int}\left(\Pi^{n}(D)\right)$. From this it would follow that $X$ has either an attracting periodic orbit or a repelling periodic orbit (Lemma 4.1). This contradicts the assumption and the proof follows. The Corollary will follow easily from the Theorem.

In $\S 2$ we describe the concepts used above. In $\S 3$ we prove some useful lemmas. In $\S 4$ we prove the Theorem and the Corollary.

\section{Preliminary Results}

In what follows, $M$ denotes a closed 3-manifold and $X=X_{t}$ denotes a flow in $M$. We always assume that $X$ is orientable and still denote by $X$ the corresponding vector field (assumed to be $C^{1}$ at least). Given a subset $D$ we denote by $\partial D, \operatorname{Cl}(D)$ and $\operatorname{int}(D)$ the boundary, the closure and the interior of $D$ respectively. An orbit of $X$ is a set $O=O_{X}(q)=\left\{X_{t}(q): t \in \mathbb{R}\right\}$ for some $q \in M$. It follows that a closed orbit $O=O_{X}(q)$ of $X$ is either singular $(X(q)=0)$ or periodic $\left(X_{t}(q)=q\right.$ for some $t>0$ minimal). If $O$ is an orbit of $X$, we define

$$
W_{X}^{s}(O)=\left\{q \in M: \lim _{t \rightarrow \infty} d\left(X_{t}(q), O\right)=0\right\}
$$

and

$$
W_{X}^{u}(O)=\left\{q \in M: \lim _{t \rightarrow-\infty} d\left(X_{t}(q), O\right)=0\right\} .
$$

We shall call $W_{X}^{s}(O)$ (resp. $\left.W_{X}^{u}(O)\right)$ the stable (resp. unstable) set of $O$.

The $\omega$-limit set of $p \in M, \omega_{X}(p)$, is the set of $x \in M$ for which there is a sequence $t_{n} \rightarrow \infty$ such that $x=\lim _{n \rightarrow \infty} X_{t_{n}}(p)$. The $\alpha$-limit set of $p$ is $\alpha_{X}(p)=\omega_{-X}(p)$. A compact invariant set $B$ of $X$ is transitive if $B=\omega_{X}(p)$ for some $p \in B$. The flow $X$ is transitive if $M$ is a transitive set.

The nonwandering set of $X, \Omega(X)$, is the set of $p \in M$ such that for every neighborhood $U$ of $p$ and $T>0$, there is $t>T$ satisfying $X_{t}(U) \cap U \neq \emptyset$. 
A compact invariant set $\Lambda$ of $X$ is isolated if there is a compact neighborhood $U$ of $\Lambda$ such that

$$
\Lambda=\bigcap_{t \in \mathbb{R}} X_{t}(U) .
$$

An isolated set is attracting if there is $U$ as above such that $X_{t}(U) \subset U$ for all $t>0$, and repelling if it is attracting for the reversed flow $-X$. An attractor of $X$ is a transitive attracting set and a repeller is a transitive repelling set.

A compact invariant set $H$ of $X$ is hyperbolic if there is a continuous invariant tangent bundle decomposition $T M / H=E_{H}^{s} \oplus E_{H}^{X} \oplus E_{H}^{u}$ such that $E_{H}^{X}$ is the direction of the flow and, for some constants $\lambda>0, C>1$ it follows that

1) $\left\|D X_{t} / E_{H}^{s}\right\| \leq C^{-1} e^{-t \lambda}$, for every $t \geq 0$;

2) $\left\|D X_{t} / E_{H}^{u}\right\| \geq C e^{t \lambda}$, for every $t \geq 0$.

The Stable Manifold Theorem [HPS] asserts that if $X$ is $C^{r}$ and $p \in H$, then

$$
W_{X}^{s s}(p)=\left\{q \in M: \lim _{t \rightarrow \infty} d\left(X_{t}(q), X_{t}(p)\right)=0\right\}
$$

and

$$
W_{X}^{u u}(p)=\left\{q \in M: \lim _{t \rightarrow-\infty} d\left(X_{t}(q), X_{t}(p)\right)=0\right\}
$$

are $C^{r}$ immersed submanifolds of $M$. In particular,

$$
W_{X}^{s}(p)=W_{X}^{s}\left(O_{X}(p)\right)=\bigcup_{t \in \mathbb{R}} W_{X}^{s s}\left(X_{t}(p)\right)
$$

and

$$
W_{X}^{u}(p)=W_{X}^{u}\left(O_{X}(p)\right)=\bigcup_{t \in \mathbb{R}} W_{X}^{u u}\left(X_{t}(p)\right)
$$

are also $C^{r}$ submanifolds of $M$. A closed orbit is hyperbolic if it is hyperbolic as a closed invariant set.

A hyperbolic set is a basic set if it is transitive and isolated. We observe that a basic set either is a singularity or else has no singularities and is the closure of its periodic orbits.

We say that $X$ is Axiom $A$ if $\Omega(X)$ is hyperbolic and the closure of the closed orbits. It follows from the Spectral Decomposition Theorem [S] that if $X$ has an Axiom A flow, then $X$ exhibits a finite disjoint collection of basic sets $\Lambda_{1}, \cdots, \Lambda_{s}$ such that

$$
\Omega(X)=\Lambda_{1} \cup \cdots \cup \Lambda_{s} .
$$

A flow $X$ on $M$ is Anosov if $M$ is a hyperbolic set of $X$. An Anosov flow is Axiom A but not conversely.

A hyperbolic strange attractor of a flow $X$ is a hyperbolic attractor $\Lambda$ of $X$ satisfying $\operatorname{dim}\left(E^{u} / \Lambda\right)=1$. Similarly, a hyperbolic strange repeller is a hyperbolic repeller $\Lambda$ satisfying $\operatorname{dim}\left(E^{s} / \Lambda\right)=1$.

All the definitions and facts above hold for diffeomorphisms $[\mathbf{S}]$.

A surface will be a closed embedded 2-manifold $S$ in $M$. We say that $S$ is transverse to $X$ whenever $X(p) \notin T_{p} S$ for every $p \in S$. If $S$ is a surface transverse to $X$, we define

$$
\sigma_{S}=\left\{q \in M: O_{X}(q) \cap S=\emptyset\right\} .
$$

Clearly $\sigma_{S}$ is a compact invariant isolated set of $X$. Denote by

$$
\Pi: \operatorname{Dom}(\Pi) \subset S \rightarrow S
$$


the return map induced by $X$ on $S$, where $\operatorname{Dom}(\Pi)$ is the domain of $\Pi$. We denote by $\operatorname{Im}(\Pi)=\Pi(\operatorname{Dom}(\Pi))$ the image of $\Pi$.

We finish this section with two examples.

Example 2.1. Let $L$ be the Anosov flow described in $[\mathrm{BL}$. Then, $L$ has a transverse torus $T$ and and a unique periodic orbit $O$ that does not intersect $T$. Choose a periodic orbit $O^{\prime} \neq O$. By DA surgery [BW, p. 9] on $O^{\prime}$, we can construct an Axiom A flow $X$ so that $T$ is transverse to $X, O^{\prime}$ is an attracting periodic orbit of $X$ (in particular, $X$ is not transitive) and $O$ is the unique orbit of $X$ that does not intersect $T$. This example shows that the conclusion of the Corollary is false for general Axiom A flows (instead of Anosov ones).

Example 2.2. Here we show that the condition $\Omega(X) \cap T \neq \emptyset$ does not imply the transitivity of Anosov flows $X$ with a transverse torus $T$. Let $L, T$ and $O$ be as in the previous example. Denote by $M_{1}$ the supporting manifold of $L$. By DA surgery on $O$ we can construct an Axiom A flow $X^{1}$ so that $T$ is transverse to $X^{1}$ and $X^{1}$ has a repelling periodic orbit $O_{1}$. Now, consider a solid torus neighborhood $S T_{1}$ around $O_{1} . X^{1}$ is transverse to the torus boundary $T_{1}$ of $S T_{1}$ and points inward to $M_{1} \backslash \operatorname{int}\left(S T_{1}\right)$ in $T_{1}$. Define $X^{2}=-X^{1}$ and let $M_{2}$ be the supporting manifold of $X^{2}$. It follows that $X^{2}$ has an attracting periodic orbit, which we denoted by $O_{2}$. Choose a solid torus neighborhood $S T_{2}$ around $O_{2}$ so that $X$ points outward to $S T_{2}$ in the torus boundary $T_{2}$ of $S T_{2}$. We denote $M_{i}^{\prime}=M_{i} \backslash \operatorname{int}\left(S T_{i}\right)$ for $i=1,2$. Gluing $M_{1}^{\prime}$ to $M_{2}^{\prime}$ with a suitable diffeomorphism from $T_{1}=\partial M_{1}^{\prime}$ to $T_{2}=\partial M_{2}^{\prime}$ as in FW, $\S 2$ p. 162], we can construct an Anosov flow $X$ satisfying:

(1) $T$ is a transverse torus of $X$,

(2) $X$ is not transitive and

(3) $\Omega(X) \cap T \neq \emptyset$.

\section{USEFUL LEMMAS}

We start with a short description of $C^{1}$ diffeomorphisms on regions of $S^{2}$, the 2 -sphere. By a region of $S^{2}$ we mean a proper closed submanifold $R$ of $S^{2}$. It follows that $R$ is obtained from a closed disk $D$ in $S^{2}$ by removing a finite collection of open disks in $D$ (these are the holes of $R$ ). More precisely, there are $s(R) \in \mathbb{N}$ and a disjoint collection of simple closed curves $\gamma_{0}(R), \cdots, \gamma_{s(R)}(R) \subset S^{2}$ such that

- $\gamma_{i}(R)$ bounds a closed disk $D_{i}(R) \subset S^{2}$ and if $s(R) \geq 1$, then $D_{i}(R) \subset$ $\operatorname{int}\left(D_{0}(R)\right), \forall i \in\{1, \cdots, s(R)\}$;

- if $s(R) \geq 1$, then $D_{i}(R) \cap D_{j}(R)=\emptyset, \forall i \neq j \in\{1, \cdots, s(R)\}$;

- $R$ is either $D_{0}(R)$ (if $s(R)=0$ ) or $D_{0}(R) \backslash\left(\bigcup_{i=1}^{s(R)} D_{i}(R)\right)$ (otherwise).

Under such notation we have the following lemma. It seems to be well known and we prove it here for completeness.

Lemma 3.1. Let $U$ be a region of $S^{2}$ and $f: U \rightarrow f(U) \subset \operatorname{int}(U)$ be a $C^{1}$ diffeomorphism. If

$$
\Lambda^{*}=\bigcap_{n=0}^{\infty} f^{n}(\operatorname{int}(U))
$$


then there is a region $U^{*} \subset U$ of $S^{2}$ satisfying:

1) $f\left(U^{*}\right) \subset \operatorname{int}\left(U^{*}\right)$;

2) $\Lambda^{*}=\bigcap_{n=0}^{\infty} f^{n}\left(\operatorname{int}\left(U^{*}\right)\right)$;

3) if $s\left(U^{*}\right) \geq 1, f\left(\gamma_{i}\left(U^{*}\right)\right)$ is not null-homotopic in $U^{*}, \forall i \in\left\{1, \cdots, s\left(U^{*}\right)\right\}$.

Proof. Let $\mathcal{R}$ be the collection of all regions $R \subset U$ of $S^{2}$ such that $f(R) \subset \operatorname{int}(R)$ and $\Lambda^{*}=\bigcap_{n=0}^{\infty} f^{n}(\operatorname{int}(R))$. Clearly $\mathcal{R} \neq \emptyset$ since $U \in \mathcal{U}$. Then there is $U^{*} \in \mathcal{R}$ such that $s\left(U^{*}\right) \leq s(R)$ for every $R \in \mathcal{R}$.

Let us prove that $U^{*}$ satisfies properties (1)-(3). Indeed, (1) and (2) hold by the definition of $\mathcal{R}$. To prove (3) we assume $s\left(U^{*}\right) \geq 1$ and denote $s=s\left(U^{*}\right)$, $\gamma_{i}=\gamma_{i}\left(U^{*}\right), D_{i}=D_{i}\left(U^{*}\right)$ for $i \in\{1, \cdots, s\}$.

Suppose by contradiction that (3) fails, i.e., there is $i_{0} \in\{1, \cdots, s\}$ such that $f\left(\gamma_{i_{0}}\right)$ is null-homotopic in $U^{*}$. Then $f\left(\gamma_{i_{0}}\right)$ bounds a closed disk $D \subset U^{*}$. Define

$$
W^{*}=f\left(U^{*}\right) \cup D \text {. }
$$

We have the following properties.

Claim 3.2. $W^{*}$ is a region in $S^{2}$ and $s\left(W^{*}\right)<s$.

Proof. Since $f$ is a diffeomorphism, we have that $f\left(U^{*}\right)$ is a region of $S^{2}$ with $\partial f\left(U^{*}\right)=f\left(\partial U^{*}\right)$. In particular, $s\left(f\left(U^{*}\right)\right)=s\left(U^{*}\right)$ and $\forall i \in\{1, \cdots, s\}$ there is a unique $j \in\left\{1, \cdots, s\left(f\left(U^{*}\right)\right)\right\}$ such that $f\left(\gamma_{i}\right)=\gamma_{j}\left(f\left(U^{*}\right)\right)$.

If $f\left(\gamma_{i_{0}}\right)=\gamma_{0}\left(f\left(U^{*}\right)\right)$, then $D_{0}\left(f\left(U^{*}\right)\right)=D$ because $f\left(U^{*}\right) \subset \operatorname{int}\left(U^{*}\right)$. This implies that $W^{*}=D$ and so $W^{*}$ is a region with $s\left(W^{*}\right)=0$. Since $s \geq 1$, by assumption, we have that $s\left(W^{*}\right)<s$ in this case.

If $f\left(\gamma_{i_{0}}\right) \neq \gamma_{0}\left(f\left(U^{*}\right)\right)$, we have that $W^{*}$ is obtained by attaching the disk $D$ to $f\left(U^{*}\right)$ along $f\left(\gamma_{i_{0}}\right)$. Then, $W^{*}$ is a region with $s\left(W^{*}\right)=s-1$. Since $s-1<s$, we have that $s\left(W^{*}\right)<s$ in this case as well. This proves Claim 3.2.

Claim 3.3. $f\left(W^{*}\right) \subset \operatorname{int}\left(W^{*}\right)$.

Proof. This follows from the following inclusions,

$$
f\left(W^{*}\right)=f\left(f\left(U^{*}\right) \cup D\right) \subset f\left(\operatorname{int}\left(U^{*}\right)\right)=\operatorname{int}\left(f\left(U^{*}\right)\right) \subset \operatorname{int}\left(W^{*}\right) .
$$

This proves Claim 3.3.

Claim 3.4. $\Lambda^{*}=\bigcap_{n=0}^{\infty} f^{n}\left(\operatorname{int}\left(W^{*}\right)\right)$.

Proof. Observe that $\Lambda^{*} \subset \operatorname{int}\left(W^{*}\right)$ and so $\Lambda^{*} \subset \bigcap_{n=0}^{\infty} f^{n}\left(\operatorname{int}\left(W^{*}\right)\right)$ because $f\left(\Lambda^{*}\right)=$ $\Lambda^{*}$. On the other hand, since $\operatorname{int}\left(W^{*}\right) \subset U^{*}$, we have

$$
\bigcap_{n=0}^{\infty} f^{n}\left(\operatorname{int}\left(W^{*}\right)\right) \subset \bigcap_{n=0}^{\infty} f^{n}\left(\operatorname{int}\left(U^{*}\right)\right)=\Lambda^{*}
$$

since $U^{*} \in \mathcal{U}$. So $\Lambda^{*}=\bigcap_{n=0}^{\infty} f^{n}\left(\operatorname{int}\left(W^{*}\right)\right)$. This proves Claim 3.4.

The above claims imply $W^{*} \in \mathcal{R}$ and $s\left(W^{*}\right)<s=s\left(U^{*}\right)$, a contradiction since $R \in \mathcal{R} \rightarrow s(R)$ is minimal at $R=U^{*}$. Then property (3) holds. The proof of Lemma 3.1 is completed.

The lemma below is straightforward. Recall that a hyperbolic periodic orbit $O$ is saddle-type if it satisfies $\operatorname{dim} E^{s}=\operatorname{dim} E^{u}=1$.

Lemma 3.5. Let $X$ be a flow with a transverse surface $S$ on $M$. If $\sigma_{S}$ is a hyperbolic saddle-type periodic orbit of $X$, then there are simple closed curves $C_{i}^{j} \subset$ 


$$
\begin{aligned}
S \cap W_{X}^{j}\left(\sigma_{S}\right) & (i=1,2, j=u, s) \text { such that } \\
& \operatorname{Dom}(\Pi)=S \backslash\left(C_{1}^{s} \cup C_{2}^{s}\right) \text { and } \operatorname{Im}(\Pi)=S \backslash\left(C_{1}^{u} \cup C_{2}^{u}\right) .
\end{aligned}
$$

Proof. We shall construct $C_{i}^{s}(i=1,2)$ since the construction of $C_{i}^{u}$ can be done by applying the same argument to $-X$. Assume that $\sigma_{S}$ is a hyperbolic saddletype periodic orbit of $X$ and fix $p \in \sigma_{S}$. Then, both $W_{X}^{s s}(p)$ and $W_{X}^{u u}(p)$ are one-dimensional.

Denoting by $t_{0} \in \mathbb{R}^{+}$the period of $\sigma_{S}$, we have $X_{t_{0}}\left(W_{X}^{\gamma}(p)\right)=W_{X}^{\sigma}(p)$ for $\gamma=s s, u u$. In particular, $W_{X}^{s}(p)$ is either a cylinder or a Möbius band provided $X_{t_{0}} / W^{s}(p)$ is orientation-preserving or orientation-reversing (resp.). Similarly for $W_{X}^{u}(p)$.

A closed interval in $W_{X}^{s s}(p)$ is called a fundamental domain if its boundary points are $a$ and $b=X_{t_{0}}(a)$ (in the orientation-preserving case) or else $a$ and $b=X_{2 t_{0}}(a)$ (in the orientation-reversing case). It is clear that $p$ does not belong to any fundamental domain.

Now, take a fundamental domain $I$ of $W_{X}^{s s}(p)$ sufficiently close to the orbit of $p$. Clearly $\forall q \in I$ the backward orbit of $q$ meets $S$. If we denote by $\pi$ the backward first intersection map induced by $X$ from $I$ to $S$, we have that $\pi(a)=\pi(b)$. In particular, $\mathcal{C}_{1}^{s}=\pi(I)$ is a simple closed curve in $S$.

Note that in the orientation-preserving case we can find a fundamental domain in $W_{X}^{s s}(p)$ different from $I$. It is such a fundamental domain that produces the simple closed curve $\mathcal{C}_{2}^{s}$ (for this we just consider its first intersection map as before).

It is not difficult to see that $\operatorname{Dom}(\Pi)=S \backslash\left(\mathcal{C}_{1}^{s} \cup \mathcal{C}_{2}^{s}\right)$ as claimed in the lemma. Indeed, the forward orbits starting at $S$ and not returning to $S$ are necessarily contained in $W_{X}^{s}(p)$. So, such orbits cross every fundamental domain of $W_{X}^{s s}(p)$ once. This completes the proof.

Proposition 3.6. Let $X$ be an Axiom A flow with a transverse surface $S$ on $M$. If $X$ has a unique orbit that does not intersect $S$, then $\sigma_{S}$ is a hyperbolic saddle-type periodic orbit.

Proof. First we prove that $\sigma_{S}$ is neither an attracting nor a repelling set. Indeed, suppose that $\sigma_{S}$ is attracting and define

$$
l_{S}=\left\{x \in S: X_{t}(x) \cap S=\emptyset, \forall t>0\right\} .
$$

Clearly $l_{S}$ is a closed subset of $S . l_{S}$ is not empty. Indeed, consider a neighborhood $U$ of $\sigma_{S}$ such that $X_{t}(U) \subset U(t>0)$ and

$$
\sigma_{S}=\bigcap_{t>0} X_{t}(U) .
$$

This neighborhood exists because $\sigma_{S}$ is an attracting set. Clearly we can choose $U$ close enough to $\sigma_{S}$ such that

$$
U \cap S=\emptyset .
$$

Since $U$ is open and $\sigma_{S}$ is closed, there is $x \in U \backslash \sigma_{S}$. Since $U \cap S=\emptyset, X_{t}(U) \subset U$ for $t>0$ and $x \in U$, we have that $X_{t}(x) \notin S$ for every $t>0$. Then, since $x \notin \sigma_{S}$, it follows that there is a first $t_{x}>0$ such that $y=X_{-t_{x}}(x) \in S$. Note that $X_{t}(y) \notin S$ for every $t \in\left(0, t_{x}\right]$ by the definition of $t_{x}$. In addition, $X_{t}(y)=X_{t-t_{x}}(x) \in U$, and so, $X_{t}(y) \notin S$ for every $t>t_{x}$. This proves that $X_{t}(y) \notin S$ for every $t>0$ proving $y \in l_{S} \neq \emptyset$. Since $\sigma_{S}$ is an attracting set, we conclude that $l_{S}$ is a nonempty open and closed set of $S$. Since $S$ is connected we conclude that $l_{S}=S$. We claim that $\Omega(X) \subset \sigma_{S}$. Indeed, suppose by contradiction that there is $x \in \Omega(X) \backslash \sigma_{S}$. Since 
$x \notin \sigma_{S}$, it follows that there is a point $y$ in the orbit of $x$ belonging to $S$. Since $\Omega(X)$ is invariant, $y \in \Omega(X)$. But $l_{S}=S$ and so there is a first $t_{y}>0$ such that $X_{t_{y}}(y) \in U$. It follows that there is a neighborhood $V$ of $y$ such that $X_{t_{y}}(V) \subset U$. This is a contradiction since $y \in \Omega(X), X_{t}(U) \subset U(t>0)$ and $U \cap S=\emptyset$. This proves $\Omega(X) \subset \sigma_{S}$. Now, let $x \in S$ and $y \in \alpha_{X}(x)$. It follows that $y \in \Omega(X)$ and so $y \in \sigma_{S}$. But this is impossible since $\sigma_{S}$ is attracting and $x \notin S$. This proves that $\sigma_{S}$ cannot be attracting. The same argument applied to $-X$ proves that $\sigma_{S}$ cannot be a repelling set.

Second we prove that $\sigma_{S}$ is not a hyperbolic singularity. For this, suppose by contradiction that $\sigma_{S}$ is. Since $\sigma_{S}$ is neither attracting nor repelling, we have that $\sigma_{S}$ is saddle-type. Using $\operatorname{dim}(M)=3$ we have that $\operatorname{dim}\left(W_{X}^{s}\left(\sigma_{S}\right)\right)=2$ and $\operatorname{dim}\left(W_{X}^{u}\left(\sigma_{S}\right)\right)=1$ by reversing the flow direction if necessary. As in the proof of Lemma 3.5, one sees that $\operatorname{Dom}(\Pi)=S \backslash C^{s}$ for some simple closed curve $C^{s} \subset W_{X}^{s}\left(\sigma_{S}\right)$ (recall that $\Pi$ is the return map). Moreover, recalling that $\operatorname{Im}(\Pi)=\Pi(\operatorname{Dom}(\Pi))$ denotes the image of $\Pi$, we have that $\operatorname{Im}(\Pi)=S \backslash\{$ two points\}. In particular, $\mathrm{Cl}(\operatorname{Im}(\Pi))=S$. Analyzing the return map in a linear flow box around $\sigma_{s}$ given by [dMP, Grobman-Hartman Theorem] we would have that either $\mathrm{Cl}(\operatorname{Im}(\Pi))$ contains an embedded $S^{2}$ (if $C^{s}$ is null-homotopic in $S$ ) or $\mathrm{Cl}(\operatorname{Im}(\Pi))$ is diffeomorphic to the closed surface $S^{\prime}$ obtained by cutting $S$ open along $C^{s}$ and capping the resulting circle boundaries with 2-disks (otherwise). The first case would imply that $S=\mathrm{Cl}(\operatorname{Im}(\Pi))$ is disconnected, a contradiction. The second case would imply that $S$ is diffeomorphic to $S^{\prime}$, again a contradiction. These contradictions show that $\sigma_{S}$ is not a hyperbolic singularity.

Finally, we prove the proposition. Since $X$ has a unique orbit that does not intersect $S$, we have that $\sigma_{S}$ is a closed orbit, and so, $\sigma_{S}$ is either periodic or singular. Since $X$ is Axiom A, we have that $\sigma_{S}$ is hyperbolic. Since $\sigma_{S}$ is not a hyperbolic singularity, we have that $\sigma_{S}$ is a hyperbolic periodic orbit. Since $\sigma_{S}$ is neither attracting nor repelling, we have that $\sigma_{S}$ is saddle-type. This finishes the proof.

\section{Proof of the Theorem and the Corollary}

We start by proving a lemma dealing with the existence of attracting or repelling periodic orbits for Axiom A flows with a transverse surface.

Lemma 4.1. Let $X$ be an Axiom A flow with a transverse surface $S$. If there are a disk $D \subset S$ and an integer $n \geq 1$ satisfying:

1) $D, \Pi(D), \Pi^{2}(D), \cdots, \Pi^{n-1}(D) \subset \operatorname{Dom}(\Pi)$;

2) $\Pi^{i}(D) \cap \Pi^{j}(D)=\emptyset$, for $i \neq j \in\{1, \cdots, n-1\}$;

3) $D \subset \operatorname{int}\left(\Pi^{n}(D)\right)$,

then $X$ has either an attracting periodic orbit or a repelling periodic orbit.

Proof. Recall that $\Pi$ denotes the return map induced by $X$ in $S$. Denote by $\Pi^{-1}$ the inverse of $\Pi$. Define $U=\Pi^{n}(D)$ and $F=\Pi^{-n} / U$. Then, $U$ is a disk, $F$ is a diffeomorphism and $F: U \rightarrow F(U)=D \subset \operatorname{int}(U)$ by the hypothesis (3). Similarly, the inverse $G=\Pi^{n} / D: D \rightarrow G(D)=U$ of $F$ is a diffeomorphism.

Next, we consider two copies $U_{1}, U_{2}$ of $U$ and two copies $D_{1}, D_{2}$ of $D$. Note that $F: U_{1} \rightarrow D_{1}$ and $G: D_{2} \rightarrow U_{2}$. Define $A_{1}=U_{1} \backslash D_{1}$ and $A_{2}=U_{2} \backslash D_{2}$. Both $A_{1}$ and $A_{2}$ are annuli, and so, they are diffeomorphic. Then $S^{2}=U_{1} \cup_{\phi} U_{2}$ for a suitable diffeomorphism $\phi: A_{1} \rightarrow A_{2}$. Using $\phi$ we can construct a diffeomorphism 
$H: S^{2}=U_{1} \cup_{\phi} U_{2} \rightarrow S^{2}$ such that $H / U_{1}=F, H / D_{2}=G$ and $\Omega(H)=\Omega(F) \cup \Omega(G)$. But $\Omega(F)=\Omega(X) \cap U_{1}$ since $F$ is the return map induced by $-X$. Since $X$ is Axiom A, we conclude that $\Omega(F)$ is hyperbolic and the closure of its periodic points. Similarly, $\Omega(G)$ is hyperbolic and the closure of its periodic points. Then $H$ is an Axiom A diffeomorphism. By [Pl, Corollary 1] we have that $H$ has either an attracting or a repelling periodic point $x$. Since both $F$ and $G$ are flow defined, it follows that $x$ belongs to an attracting or a repelling periodic orbit of $X$. This proves the result.

Next we remember the definition of essential orbit given in the Introduction.

Definition 4.2. Let $X$ be a flow and $S$ a surface transverse to $X$. An orbit $O$ of $X$ is essential for $S$ if there is no simple closed null-homotopic curve in either $W_{X}^{s}(O) \cap S$ or $W_{X}^{u}(O) \cap S$.

Proof of the Theorem. Let $X, T$ and $O$ be as in the statement of the theorem. By Proposition 3.6, we have that $\sigma_{S}$ is a hyperbolic saddle-type periodic orbit. In particular, $X$ has no singularities. By Lemma 3.5, there are simple closed curves $C_{i}^{j} \subset T \cap W_{X}^{j}\left(\sigma_{T}\right)(i=1,2, j=s, u)$ such that

$$
\operatorname{Dom}(\Pi)=S \backslash\left(C_{1}^{s} \cup C_{2}^{s}\right) \text { and } \operatorname{Im}(\Pi)=S \backslash\left(C_{1}^{u} \cup C_{2}^{u}\right) .
$$

There are two cases, namely, $C_{1}^{s}$ and $C_{2}^{s}$ are either equal or disjoint. We shall assume the latter case since the former one is simpler.

Assume that the hypothesis $(*)$ below holds:

(*) $X$ has neither attracting periodic orbits nor repelling periodic orbits.

To prove the theorem we have to prove that $X$ is transitive. To prove that $X$ is transitive we assume by contradiction that it is not. Then there is a spectral decomposition $\Omega(X)=\Lambda_{1} \cup \cdots \cup \Lambda_{l}$ of $\Omega(X)$, where the $\Lambda_{i}$ 's are basic sets of $X$. One of these basic sets $\left(\Lambda=\Lambda_{1}\right.$ say) is an attractor and one of them is a repeller. Since $X$ has no singularities, $(*)$ implies that both $\Lambda_{1}$ and $\Lambda_{2}$ are strange. Clearly $\sigma_{T}$ belongs to (only) one of the basic sets. Then reversing the flow direction if necessary, we can assume that $\sigma_{T} \cap \Lambda=\emptyset$. Since $\Lambda$ is a hyperbolic strange attractor of $X$, we have that $\Lambda^{*}=\Lambda \cap T$ is a hyperbolic strange attractor of $\Pi$. In particular, $\operatorname{dim} E^{u} / \Lambda^{*}=1$. Replacing $\Pi$ by a power $\Pi^{n}$ if necessary we can assume that $\Pi\left(\Lambda^{*}\right)=\Lambda^{*}$. Henceforth $\Lambda^{*}$ is connected. So, there is a connected compact neighborhood $U \subset \operatorname{int}\left(T \backslash C_{1}^{s}\right)$ of $\Lambda^{*}$ such that if $f=\Pi / U$, then $f: U \rightarrow f(U) \subset \operatorname{int}(U)$ and $\Lambda^{*}=\bigcap_{n \in \mathbb{N}} f^{n}(\operatorname{int}(U))$. Note that $U \subset \operatorname{Dom}(\Pi)$.

Since $O$ is essential for $T$, we have that $C_{1}^{s}$ is not null-homotopic in $T$ (see Definition 4.2). Thus, the surface-with-boundary $A$ obtained by cutting $T$ open along $C_{1}^{s}$ is an annulus which clearly embeds into $S^{2}$. Note that $\operatorname{int}\left(T \backslash C_{1}^{s}\right) \subset \operatorname{int}(A)$ and so $U \subset \operatorname{int}(A)$. Since $A$ embeds into $S^{2}$, we have that $U$ is a proper connected subset of $S^{2}$. Using Lyapunov functions [S], we can assume that $U$ is a region of $S^{2}$. Then, by Lemma 3.1, there is a region $U^{*} \subset U$ of $S^{2}$ satisfying the properties (1)-(3) in that lemma. Following the notation at the beginning of $\S 3$, we denote $s=s\left(U^{*}\right), \gamma_{i}=\gamma_{i}\left(U^{*}\right)$ and $D_{i}=D_{i}\left(U^{*}\right)$ for $i \in\{1, \cdots, s\}$.

Since $\operatorname{dim} E^{u} / \Lambda^{*}=1$, we have by [R, Theorem 9.1, p. 304] that $s \geq 3$.

Define

$$
\Gamma=\left\{i \in\{1, \cdots, s\}: \gamma_{i} \text { bounds a disk } D(i) \subset \operatorname{Dom}(\Pi)\right\} .
$$

Then we have the following properties. 
Claim 4.3. $\Gamma \neq \emptyset$.

Proof. Since $U^{*} \subset U \subset \operatorname{Dom}(\Pi)=T \backslash\left(C_{1}^{s} \cup C_{2}^{s}\right)$, we have

$$
\gamma_{i} \cap\left(C_{1}^{s} \cup C_{2}^{s}\right)=\emptyset, \quad \forall i .
$$

On the other hand, $U^{*} \subset U \subset \operatorname{int}(A)$ and $A$ is an annulus. Since $s \geq 3$, there is $i \in\{1, \cdots, s\}$ such that $\gamma_{i}$ bounds a disk $D \subset \operatorname{int}(A)$. In particular, $D \cap C_{1}^{s}=\emptyset$. If $D \cap C_{2}^{s} \neq \emptyset$, we would have $C_{2}^{s} \subset \operatorname{int}(D)$ (otherwise $\gamma_{i} \cap C_{2}^{s} \neq \emptyset$ contradicting $\left.\gamma_{i} \cap\left(C_{1}^{s} \cup C_{2}^{s}\right)=\emptyset\right)$ and so $C_{2}^{s}$ would be null-homotopic in $T$, a contradiction since $\sigma_{T}$ is essential. So, $D \cap C_{2}^{s}=\emptyset$. Since $\operatorname{Dom}(\Pi)=T \backslash\left(C_{1}^{s} \cup C_{2}^{s}\right)$, we conclude that $D \subset \operatorname{Dom}(\Pi)$, and so, $i \in \Gamma$ since $\gamma_{i}$ bounds $D$. This proves Claim 4.3.

Claim 4.4. If $i \in \Gamma$, then $\Pi(D(i)) \subset \operatorname{Dom}(\Pi)$.

Proof. Since $f\left(U^{*}\right) \subset U^{*} \subset U \subset \operatorname{Dom}(\Pi)$, we obtain

$$
f\left(\gamma_{i}\right) \cap\left(C_{1}^{s} \cup C_{2}^{s}\right)=\emptyset, \quad \forall i
$$

Fix $i \in \Gamma$. Since $\Pi$ is a diffeomorphism, we have that $\Pi(D(i))$ is a disk in $T$ with boundary $\partial \Pi(D(i))=f\left(\gamma_{i}\right)$. If $\Pi(D(i)) \cap C_{1}^{s} \neq \emptyset$, we would have $C_{1}^{s} \subset \Pi(D(i))$ (otherwise $f\left(\gamma_{i}\right) \cap C_{1}^{s} \neq \emptyset$, contradicting $f\left(\gamma_{i}\right) \cap\left(C_{1}^{s} \cup C_{2}^{s}\right)=\emptyset$ ) and so $C_{1}^{s}$ would be null-homotopic in $T$, a contradiction since $\sigma_{T}$ is essential. So, $\Pi(D(i)) \cap C_{1}^{s}=\emptyset$. Similarly, we use $f\left(\gamma_{i}\right) \cap\left(C_{1}^{s} \cup C_{2}^{s}\right)=\emptyset$ in order to prove $\Pi(D(i)) \cap C_{2}^{s}=\emptyset$. Since $\operatorname{Dom}(\Pi)=T \backslash\left(C_{1}^{s} \cup C_{2}^{s}\right)$, we conclude that $\Pi(D(i)) \subset \operatorname{Dom}(\Pi)$. This proves Claim 4.4 .

Claim 4.5. If $i \in \Gamma$, then $\exists j \in \Gamma$ such that $D(j) \subset \operatorname{int}(\Pi(D(i)))$.

Proof. Fixing $i \in \Gamma$ we have that $D^{\prime}=\Pi(D(i))$ is a disk and $D^{\prime} \subset \operatorname{Dom}(\Pi)$ by Claim 4.4. Since $\operatorname{Dom}(\Pi) \subset \operatorname{int}(A)$, we conclude that $D^{\prime} \subset A$. Note that $\partial D^{\prime}=$ $f\left(\gamma_{i}\right)$. In addition, $D^{\prime}$ is not contained in $U^{*}$ for otherwise $f\left(\gamma_{i}\right)$ would be nullhomotopic in $U^{*}$ contradicting the property (3) of Lemma 3.1. So, $\exists j \in\{1, \cdots, s\}$ such that $\gamma_{j} \cap D^{\prime} \neq \emptyset$. Since $\partial D^{\prime}=f\left(\gamma_{i}\right) \subset \operatorname{int}\left(U^{*}\right)$, we conclude that $\gamma_{j} \subset \operatorname{int}\left(D^{\prime}\right)$ (otherwise $\gamma_{j} \cap f\left(\gamma_{i}\right) \neq \emptyset$ contradicting $f\left(\gamma_{i}\right) \subset \operatorname{int}\left(U^{*}\right)$ ). Since $D^{\prime}$ is a disk and $\gamma_{j} \subset D^{\prime}$ is a simple closed curve, we have that $\gamma_{j}$ bounds a disk $D^{\prime \prime} \subset D^{\prime}=\Pi(D(i))$. Since $D^{\prime} \subset \operatorname{Dom}(\Pi)$, we conclude that $D^{\prime \prime} \subset \operatorname{Dom}(\Pi)$. Since $D^{\prime \prime} \subset \operatorname{Dom}(\Pi)$ and $\gamma_{j}$ bounds $D^{\prime \prime}$, we conclude that $j \in \Gamma$ and $D(j)=D^{\prime \prime} \subset \operatorname{int}(\Pi(D(i)))$. This proves Claim 4.5.

Let us finish the proof of the Theorem using the above claims. By Claim 4.3 we can choose $i_{1} \in \Gamma$. By Claim 4.4 we have that $\Pi\left(D\left(i_{1}\right)\right) \subset$ Dom( $(\Pi)$. By Claim $4.5 \exists i_{2} \in \Gamma$ such that $D\left(i_{2}\right) \subset \operatorname{int}\left(\Pi\left(D\left(i_{1}\right)\right) \subset \operatorname{Dom}(\Pi)\right.$ and so on. In this way we construct a sequence $i_{1}, i_{2}, \ldots, i_{l} \in \Gamma$ such that

$$
D\left(i_{k+1}\right) \subset \operatorname{int}\left(\Pi\left(D\left(i_{k}\right)\right)\right.
$$

for every $k=1, \cdots, l-1$. Since $\Gamma$ is finite, we can assume that $l$ is the first positive integer such that $i_{1}=i_{l}$. It follows that $D=D_{i_{1}}$ and $n=l-1$ satisfy the hypotheses (1)-(3) of Lemma 4.1. Since $X$ is Axiom A, we conclude by this lemma that $X$ has either an attracting periodic orbit or a repelling periodic orbit, a contradiction by the hypothesis $\left({ }^{*}\right)$. This proves that $X$ is transitive and the result follows.

Proof of the Corollary. Let $X$ be an Anosov flow with a transverse torus $T$ on a closed 3-manifold. Suppose that $X$ exhibits a unique periodic orbit that does not intersect $T$. Let us prove that $X$ and $T$ satisfy the hypothesis of the Theorem. 
First we show that $X$ has a unique orbit that does not intersect $T$. For this, denote by $O$ the unique periodic orbit of $X$ that does not intersect $T$. It suffices to show $\sigma_{T}=O$. Indeed $O \subset \sigma_{T}$ by the definition of $\sigma_{T}$. To prove $\sigma_{T} \subset O$ we assume by contradiction it is not true. Then there is $q \in \sigma_{T} \backslash O$. Denote by $\Omega\left(X / \sigma_{T}\right)$ the nonwandering set of $X$ restricted to $\sigma_{T}$. Since $\sigma_{T}$ is isolated, it follows that $\Omega\left(X / \sigma_{T}\right)$ is a finite disjoint union of basic sets (Spectral Decomposition Theorem). Observe that a basic set either is a singularity or else has no singularities and is the closure of its periodic orbits. Since $X$ is Anosov, we have that $X$ has no singularities. We conclude that $\Omega\left(X / \sigma_{T}\right)=O$ and so $\lim _{t \rightarrow \pm \infty} X_{t}(q)=O$. In particular,

$$
q \in\left(W_{X}^{s}(O) \cap W_{X}^{u}(O)\right) \backslash O
$$

i.e., $O_{X}(q)$ is a homoclinic orbit of $X$ associated to $O$. Note that $O_{X}(q)$ is transversal since $X$ is Anosov. It follows that $H=\mathrm{Cl}\left(O_{X}(q)\right)$ is a hyperbolic set of $X$ containing $O$. Using $O_{X}(q) \subset H$ we can construct a periodic $\epsilon$-pseudo-orbit $Q \subset H$ containing $q$ such that the end points of $Q$ are close to $O$. In particular, $Q \cap T=\emptyset$. By the Shadowing Lemma for Flows $[\mathrm{KH}$, Theorem 18.1.6, p. 569] applied to the hyperbolic set $H$, we can find a periodic orbit $O^{\prime}$ that both shadows $Q$ and passes close to $q$. Since $Q \cap T=\emptyset$ we have that $O^{\prime} \cap T=\emptyset$ also. But $O$ is the unique periodic orbit of $X$ that does not intersect $X$. So $O^{\prime}=O$. Since $q$ is not in $O$ and $O^{\prime}$ passes close to $q$, we arrive at a contradiction. This contradiction proves that $\sigma_{T} \backslash O=\emptyset$, i.e., $\sigma_{T} \subset O$. Henceforth $\sigma_{T}=O$ as desired.

Second we prove that $\sigma_{T}$ is essential for $T$. Indeed, since $X$ is Anosov, we have that the set

$$
\mathcal{F}^{u}=\left\{W_{X}^{u}(x): x \in M\right\}
$$

is a continuous codimension-one nonsingular foliation of $M$. Since $X$ is transverse to $T$, we have that $F^{u}=\mathcal{F}^{u} \cap T$ is a continuous one-dimensional nonsingular foliation on $T$. Similarly for $F^{s}=\mathcal{F}^{s} \cap T$. Clearly every closed curve $\gamma$ in $W_{X}^{u}\left(\sigma_{T}\right) \cap T$ (resp. $W_{X}^{s}\left(\sigma_{T}\right) \cap T$ ) is a leaf of $F^{u}$ (resp. $F^{s}$ ). To prove that $\gamma$ is not null-homotopic in $T$, we use the following standard argument: If $\gamma$ were null-homotopic in $T$, then $\gamma$ would bound a disk $D$ in $T$. As is well known, the foliation $F^{s}$ (resp. $F^{u}$ ) restricted to $D$ is orientable. Applying [HH, Poincaré-Bendixon Theorem] we show that $F^{s}$ (resp. $F^{u}$ ) has a singularity in $D$, a contradiction. Henceforth $\gamma$ is not null-homotopic in $T$. We conclude that $\sigma_{T}$ is essential for $T$. This proves that $X$ and $T$ satisfy the hypothesis of the Theorem.

The Theorem implies that $X$ either has an attracting periodic orbit or a repelling periodic orbit or is transitive. But $X$ has neither an attracting periodic orbit nor a repelling periodic orbit for it is Anosov. So $X$ is transitive and the proof follows.

\section{REFERENCES}

[BW] Birman, J. and Williams, R. F., Knotted periodic orbits in dynamical systems II: Knot holders for fibered knots, Contemporary Math. 20 (1983), 1-60. MR 86a:58084

[BL] Bonatti, C. and Langevin R., Un example de flot d'Anosov transitif transverse à un tore et non conjugué à une suspension, Ergodic Theory and Dynamical Systems 14 (1994), 633-643. MR 95j:58129

[Br1] Brunella, M., Separating the basic sets of a nontransitive Anosov flow, Bull. London Math. Soc. 25 (1993), 487-490. MR 94g:58163

[Br2] Brunella, M., On the discrete Godbillon-Vey invariant and Dehn surgery on geodesic flows, Ann. Fac. Sci. Toulouse Math. (6) 3 (1994), no. 3, 335-344. MR 96a:58144

[dMP] de Melo, W. and Palis J., Geometric theory of dynamical systems. An introduction, Springer-Verlag, New York, Berlin (1982). MR 84a:58004 
[FW] Franks, J. and Williams, R. F., Anomalous Anosov flows, Lecture Notes in Math. 819 (1979), Springer, Berlin, 158-174. MR 82e:58078

[KH] Hasselblatt, B. and Katov, A., Introduction to the modern theory of dynamical systems, Cambridge University Press, Cambridge (1995). MR 96c:58055

[HT] Handel, M. and Thurston, W. P., Anosov flows on new three manifolds, Invent. Math. 59 (1980), no. 2, 95-103. MR 81i:58032

$[\mathrm{HH}]$ Hector, G. and Hirsch, U., Introduction to the geometry of foliations, Parts $A$ and B, Vieweg, Braunschweig (1983). MR 85f:57016

[HPS] Hirsch, M., Pugh, C., and Shub M., Invariant manifolds, Lecture Notes in Math. 583 (1977), Springer-Verlag, Berlin, New York. MR 58:18595]

[Pl] Plykin, R. V., Sources and sinks of A-diffeomorphisms of surfaces, Math. Sbornik, 94, 136, (1974), 233-253. MR 50:8608

[R] Robinson, C., Dynamical Systems. Stability, symbolic dynamics, and chaos, Studies in Advanced Mathematics. CRC Press, Boca Raton, FL (1995). MR 97e:58064

[S] Shub, M., Stabilité globale des systèmes dynamiques, Astérisque 56 (1978). MR 80c:58015

Instituto de Matematica, Universidade Federal do Rio de Janeiro, C. P. 68.530, CEP 21.945-970, Rio de Janeiro, Brazil

E-mail address: morales@impa.br 